\title{
Comparing the prediction of prostate biopsy outcome using the Chinese Prostate Cancer Consortium (CPCC) Risk Calculator and the Asian adapted Rotterdam European Randomized Study of Screening for Prostate Cancer (ERSPC) Risk Calculator in Chinese and European men
}

\author{
Rui Chen ${ }^{1} \cdot$ Jan F. M. Verbeek ${ }^{2} \cdot$ Yue Yang $^{1} \cdot$ Zijian Song $^{1} \cdot$ Yinghao Sun $^{1} \cdot$ Monique J. Roobol $^{2}$
}

Received: 17 November 2019 / Accepted: 20 March 2020

(c) Springer-Verlag GmbH Germany, part of Springer Nature 2020

\begin{abstract}
Purpose To externally validate the clinical utility of Chinese Prostate Cancer Consortium Risk Calculator (CPCC-RC) and Asian adapted Rotterdam European Randomized Study of Screening for Prostate Cancer Risk Calculator 3 (A-ERSPC-RC3) for prediction prostate cancer (PCa) and high-grade prostate cancer (HGPCa, Gleason Score $\geq 3+4$ ) in both Chinese and European populations.

Materials and methods The Chinese clinical cohort, the European population-based screening cohort, and the European clinical cohort included 2,508, 3,616 and 617 prostate biopsy-naive men, respectively. The area under the receiver operating characteristic curve (AUC), calibration plot and decision curve analyses were applied in the analysis.

Results The CPCC-RC's predictive ability for any PCa (AUC 0.77, 95\% CI 0.75-0.79) was lower than the A-ERSPC-RC3 (AUC $0.79,95 \%$ CI 0.77-0.81) in the European screening cohort $(p<0.001)$, but similar for HGPCa $(p=0.24)$. The CPCCRC showed lower predictive accuracy for any PCa (AUC 0.65, 95\% CI 0.61-0.70), but acceptable predictive accuracy for HGPCa (AUC 0.73, 95\% CI 0.69-0.77) in the European clinical cohort. The A-ERSPC-RC3 showed an AUC of 0.74 (95\% CI 0.72-0.76) in predicting any PCa, and a similar AUC of 0.74 (95\% CI 0.72-0.76) in predicting HGPCa in Chinese cohort. In the Chinese population, decision curve analysis revealed a higher net benefit for CPCC-RC than A-ERSPC-RC3, while in the European screening and clinical cohorts, the net benefit was higher for A-ERSPC-RC3.

Conclusions The A-ERSPC-RC3 accurately predict the prostate biopsy in a contemporary Chinese multi-center clinical cohort. The CPCC-RC can predict accurately in a population-based screening cohort, but not in the European clinical cohort.
\end{abstract}

Keywords Prostate cancer - Biopsy $\cdot$ Risk calculators $\cdot$ Asian populations $\cdot$ Net benefit $\cdot$ Clinical prediction model .

External validation

\section{Introduction}

Rui Chen and Jan F. M. Verbeek contributed equally.

Yinghao Sun

sunyhsmmu@126.com

$\triangle$ Monique J. Roobol

m.roobol@erasmusmc.nl

1 Department of Urology, Shanghai Changhai Hospital, Second Military Medical University, Changhai Road 168, Yangpu District, Shanghai 200433, China

2 Department of Urology, Erasmus University Medical Center, Room Na1706, PO Box 2040, 3000 CA Rotterdam, The Netherlands
Prostate cancer $(\mathrm{PCa})$ is the most prevalent malignancy in male in western countries, and account for one-quarter of new malignancy diagnoses in men in the United States [1]. The PCa incidence is lower in Asian countries [2,3]. This could be caused by racial differences and/or different screening strategies [4]. In western populations, PCa screening with a purely PSA based biopsy indication leads to unnecessary biopsies [5]. Would prostate cancer screening with a comparable PSA cut-off be applied in the Chinese population it would likely lead to even more unnecessary biopsies, 
giving the lower PCa detection rate at similar PSA levels in the Chinese population [6].

Risk calculators (RC) have been developed to aid physicians in shared decision making before taking the actual prostate biopsy and have shown to reduce unnecessary biopsies by more than 30\% [7]. However, the application of western-developed risk calculators in Asian countries might yield lower predictive performance. Recently published studies in Korean [8] and Chinese populations [9] showed that the Rotterdam European Randomized Study of Screening for Prostate Cancer Risk Calculator 3 (ERSPC-RC3) and Prostate Cancer Prevention Trial risk calculator (PCPT$\mathrm{RC}$ ) overestimate the risk of PCa. Therefore, the development of Asian-specific RCs for Asians including Chinese is desired. The Chinese Prostate Cancer Consortium Risk Calculator (CPCC-RC) was developed using multi-center clinical data from Chinese men to predict biopsy outcomes. This RC showed good predictive performance after external validation [10]. Recently, the ERSPC-RC3 was adapted to the Asian setting, showing excellent discrimination and calibration in Hong Kong-based development and external validation cohorts [11].

In this study, we assessed the generalizability and clinical usefulness of both the CPCC-RC and the Asian-adapted ERSPC-RC3 (A-ERSPC-RC3) by externally validating these RCs in a contemporary multi-center Chinese clinical cohort and a multi-center European clinical cohort. In addition, considering the lower prevalence of $\mathrm{PCa}$ in Asia, we also evaluated the performance of the CPCC-RC in the ERSPC Rotterdam population-based screening cohort. All men included in this study were biopsy naive.

\section{Methods}

\section{Study population}

This study is approved by Ethical review board of Changhai Hospital. The Chinese validation cohort included 2,508 referred men from five hospitals who were enrolled from January 2010 to December 2013. These men were not included in the development cohort of the CPCC-RC [10]. Biopsy indication was PSA $\geq 4.0 \mathrm{ng} / \mathrm{ml}$, abnormal digital rectal examination (DRE), or a suspicious lesion on transrectal ultrasound (TRUS) imaging. They received 8-12 systematic TRUS-guided biopsy cores, with no targeted biopsies were performed. Patient's characteristics were prospectively gathered; \% freePSA (freePSA/total PSA) and prostate volume was missing in 116 and 33 men, respectively. Missing data were imputed using all variables which were used in the development of both models. Imputation was executed by Multivariate Imputation by Chained Equations (MICE) and was repeated five times [12].
The European screening cohort consists of 3616 men biopsied at the first round of screening in the Rotterdam ERSPC section from November 1993 to March 2000. Biopsy indication was PSA $\geq 3.0 \mathrm{ng} / \mathrm{ml}$ or abnormal DRE finding. All patients underwent lateral sextant biopsies and extra biopsies whether a hypo-echogenic lesion was found. Detailed characteristics of this study and data management have been described previously $[5,13]$. This cohort is the development cohort of the original ERSPC-RC3.

The European clinical cohort consisted of 617 men from four different sites (Berlin, Münster, Paris, Rennes). Men were referred for further assessment based on a PSA $\geq 4.0 \mathrm{ng} / \mathrm{ml}$ and/or suspicious DRE. These men were not a part of the development of the ERSPC-RCs. They received standard clinical health care at each participating site ( $\geq 10$ cores transrectal ultrasound biopsies). Data on PSA, $\%$ freePSA, DRE, prostate volume and biopsy outcome were collected. Two men had no data on prostate volume and DRE finding; in these men the mean value of the prostate volume was used and DRE was set on normal.

\section{Outcome measurements and prediction models}

The primary endpoint was the presence of histologic adenocarcinoma on biopsy graded with Gleason score [14]. Highgrade (HG) PCa was defined as Gleason score $\geq 7$, other prostate cancers were defined as low-grade prostate cancer (LGPCa) [15]. The A-ERSPC-RC3 was adjusted for the Asian setting by changing the intercept of the constant and was validated in more than 2000 Chinese men [16]. The A-ERSPC-RC3 includes PSA, TRUS measured prostate volume, DRE and TRUS abnormality. The CPCC-RC included PSA, TRUS measured prostate volume and DRE abnormality, but no TRUS abnormality, while age and \%freePSA were included in the model.

\section{Statistical analysis}

Baseline characteristics of patients with PCa, LGPCa, and HGPCa were compared using the Mann-Whitney $U$ test for continuous data and chi-square test for categorical data. For each risk calculator, the predicted probabilities for the presence of any PCa and HGPCa were calculated. The accuracy of the prediction models was assessed in each cohort by discrimination and calibration properties. The discriminative power of the prediction models for the presence of any PCa or HGPCa versus men without PCa was determined by the area under the receiver operating characteristic curve (AUC). Thousand bootstrap samples were created to test significance between the AUCs.

For calibration, the observed percentages of men with presences of $\mathrm{PCa}$ were plotted against the predicted probability of men having PCa after dividing the predicted 
probabilities into deciles. For every decile, we calculated the 95\% CI of the observed percentage of responders. In addition, a histogram was made for the actual frequency of the predicted probabilities. The goodness-of-fit of the data to the predictions was evaluated by the Hosmer-Lemeshow test. Statistical analyses were performed in $\mathrm{R}$ statistical package, version 3.3.1. A $p$ value $<0.05$ was considered statistically significant.

\section{Results}

\section{Comparison of patients' characteristics}

Men in the Chinese cohort had a median age of 69 years (IQR 63-75) and the median number of biopsy cores of 10 (IQR 8-12). PCa was found in 857 (34\%) men and 599 (24\%) men had HGPCa. In the European screening cohort and the clinical cohort, the median age was 64 years and 65 years, respectively. PCa was present in $885(25 \%)$ and $290(47 \%)$ men, while HGPCa was present in $313(9 \%)$ and 142 (23\%) men, respectively. Furthermore, men from the Chinese cohort had a larger prostate volume and a higher PSA level (Table 1).

\section{External validation in the European screening cohort}

The CPCC-RC had slightly lower predictive ability for any prostate cancer (AUC $0.77,95 \%$ CI $0.75-0.79$ ) than the A-ERSPC-RC (AUC 0.79, 95\% CI 0.77-0.81, $p<0.01$ ). For HGPCa, similar predictive ability between the CPCC-RC (AUC 0.86, 95\% CI 0.83-0.88) and the A-ERSPC-RC (AUC $0.86,95 \%$ CI $0.84-0.89, p=0.24$ ) was found (Table 2). Calibration plots showed that the CPCC-RC would generally underestimate the risk of any $\mathrm{PCa}$ in the European screening cohort by $10-15 \%$. However, the CPCC-RC showed good calibration for HGPCa, especially at lower risk thresholds (Fig. 1a, b). Decision curve analysis showed a higher net benefit for the CPCC-RC above the risk threshold of 7.5\% for any PCa and 4\% for HGPCa in comparison to a biopsy all scenario men, (Fig. 2a, b). Net-benefit for the A-ERSPC$\mathrm{RC} 3$ was even higher than the CPCC-RC.

\section{External validation in the European clinical cohort}

Validation of the CPCC-RC in the European clinical cohort had a moderate discriminative ability in predicting any $\mathrm{PCa}$ (AUC 0.65, 95\% CI 0.61-0.70), and good discriminative ability in predicting HGPCa (AUC $0.73,95 \%$ CI $0.69-0.77$ ). The A-ERSPC-RC had a similar discrimination for any $\mathrm{PCa}$ (AUC 0.65, 95\% CI 0.60-0.69, $p=0.79$ ) and lower discrimination for HGPCa (AUC 0.69, 95\% CI 0.65-0.73, $p<0.01$ ).
Calibration analyses showed that the CPCC-RC would generally underestimate the risk of both any $\mathrm{PCa}$ and $\mathrm{HGPCa}$ in the European clinical cohort (Fig. 1c, d). Decision curve analysis for any PCa and HGPCa showed no improvement in net benefit when using the CPCC-RC or the A-ERSPC-RC in the European clinical dataset (Fig. 2c, d).

\section{External validation in the Chinese cohort}

External validation of A-ERSPC-RC3 in the Chinese clinical cohort showed an AUC of 0.74 (95\% CI 0.72-0.76) in predicting any $\mathrm{PCa}$, and a similar AUC of 0.74 (95\% CI 0.72-0.76) in predicting HGPCa. These AUC's are significantly lower than the AUC's of the CPCC-RC (0.77, 95\% CI 0.74-0.77, $p<0.001)$ and 0.77 (95\% CI 0.75-0.79, $p<0.001$ ), respectively. Calibration plots showed that the A-ERSPC-RC 3 underestimated the risk of any PCa in the risk range of $0-40 \%$ and overestimated the risk in the risk range over $60 \%$. The CPCC-RC, on the contrary, underestimated the risk of any PCa in higher risk ranges (30\%-100\%) but was well-calibrated in the risk range of $0-30 \%$ (Fig. 1e). Also for the prediction of HGPCa, the CPCC-RC was better calibrated than the A-ERSPC-RC3 in most risk ranges. The A-ERSPC-RC3 underestimated the risk of HGPCa in the risk range of $0-40 \%$ and overestimated the risk in the risk range of $60-100 \%$ (Fig. 1f). Improvement in net benefit was found for the CPCC-RC at risks of $12.5 \%$ and above for any $\mathrm{PCa}$ and $8 \%$ or more for HGPCa (Fig. 2e, f). For the A-ERSPC-RC, an improvement in net benefit was found from a risk threshold of $20 \%$ onward for any PCa and $15 \%$ for HGPCa. Higher net benefit was found for the CPCCRC than for the A-ERSPC-RC3. At a 10\% risk threshold for predicting HGPCa, the biopsy reduction is $25 \%$ for the CPCC-RC and $28 \%$ for A-ERSPC-RC. This threshold would result in missing $8 \%$ (CPCC-RC) and 10\% (A-ERSPC-RC) of the HGPCa diagnoses (Table 3). The use of CPCC-RC would avoid an additional 6.8 biopsies per 1000 men at risk compared to the A-ERSPC-RC. At the 10\% risk threshold the CPCC has a net benefit of 0.16. This implies that to avoid one unnecessary biopsy, without increasing the percentage of missed diagnoses of HGPCa, the CPCC-RC should be applied to six men.

\section{Discussion}

The A-ERSPC-RC3 which originated from the original ERSPC-RC can accurately predict the outcome of prostate biopsy in a contemporary Chinese multi-center clinical cohort [11]. However, the calibration plots showed an underestimation of the probability of detecting $\mathrm{PCa}$ on biopsy in the Chinese external validation cohort used in the current analyses. This underestimation is probably attributable to 


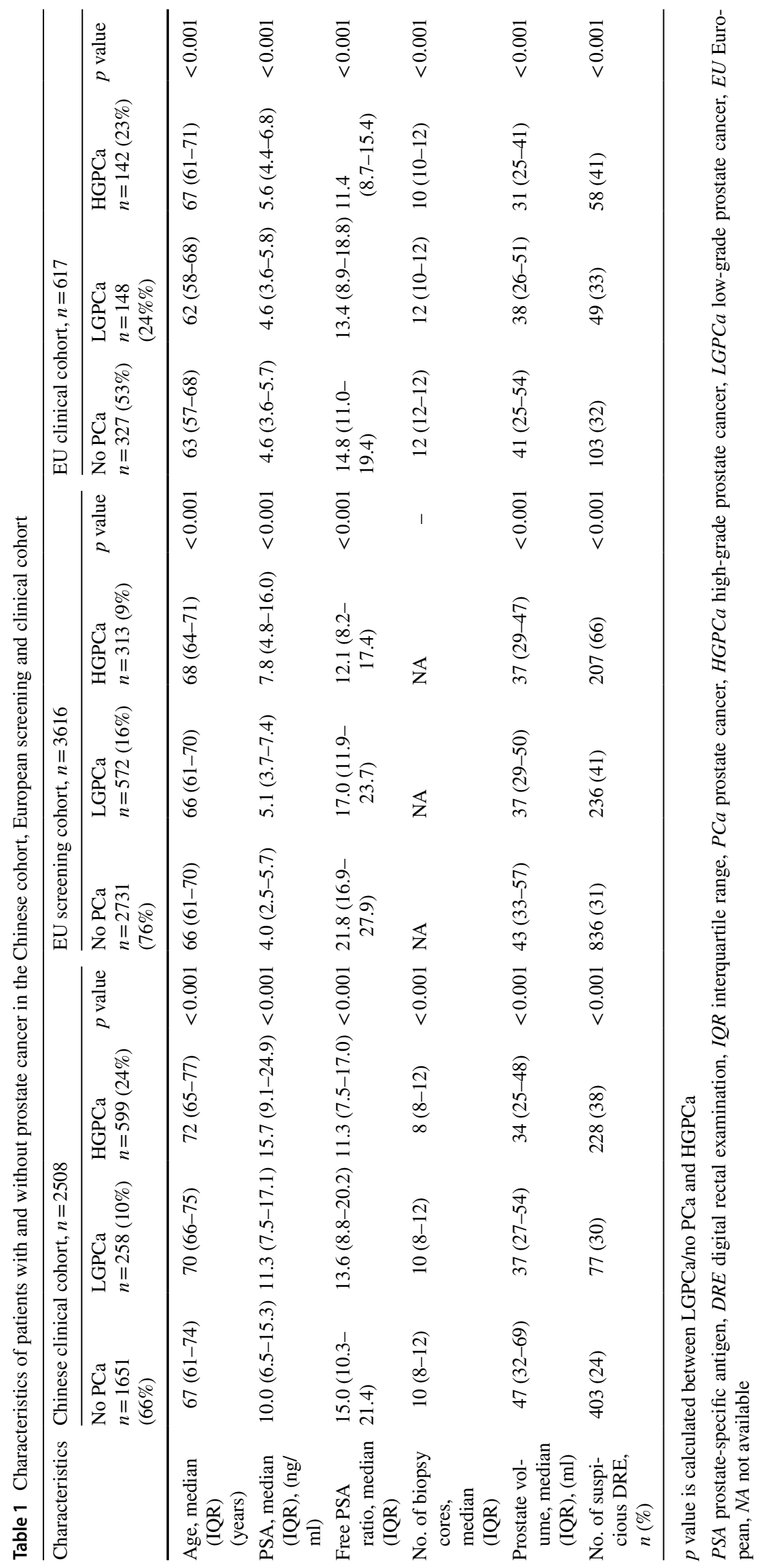


Table 2 Area under the receiver operating characteristic curve for the Chinese Prostate Cancer Consortium Risk Calculator and the Asian adapted Rotterdam European Randomized Study of Screening for Prostate Cancer Risk Calculator 3 predicting any PCa or HGPCa in the three cohorts

\begin{tabular}{lllll}
\hline Cohort-RC & Any PCa $(95 \%$ CI $)$ & $p$ value & HGPCa $(95 \%$ CI $)$ & $p$ value \\
\hline EU screening & & & $0.86(0.83-0.88)$ & \\
CPCC-RC & $0.77(0.75-0.79)$ & & $0.86(0.84-0.89)$ & 0.24 \\
A-ERSPC-RC3 & $0.79(0.77-0.81)$ & $<0.01$ & $0.73(0.69-0.77)$ & \\
EU clinical & & & $0.69(0.65-0.73)$ & $<0.01$ \\
CPCC-RC & $0.65(0.61-0.70)$ & 0.79 & & \\
A-ERSPC-RC3 & $0.65(0.60-0.69)$ & & $0.77(0.75-0.79)$ & $<0.01$ \\
Chinese & & & $0.74(0.72-0.76)$ & \\
CPCC-RC & $0.77(0.74-0.77)$ & $<0.01$ & & \\
A-ERSPC-RC3 & $0.74(0.72-0.76)$ & & & \\
\hline
\end{tabular}

$P C a$ prostate cancer, $H G P C a$ high-grade prostate cancer, EU European, $C P C C$ - $R C$ Chinese Prostate Cancer Consortium Risk Calculator, A-ERSPC-RC3 Asian adapted Rotterdam European Randomized Study of Screening for Prostate Cancer Risk Calculator 3

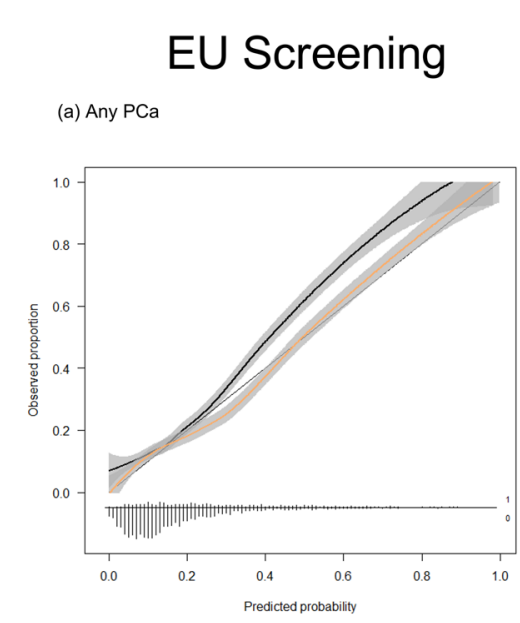

(b) HGPCa

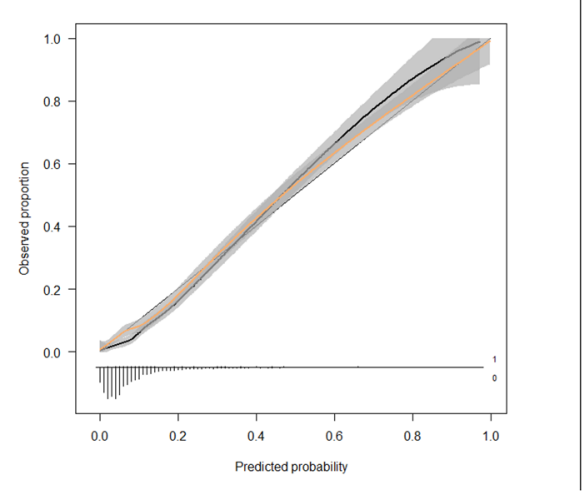

CPCC-RC

A-ERSPC-RC3

Fig. 1 Calibration plot for a predicting any prostate cancer in the European screening cohort, $\mathbf{b}$ predicting high-grade prostate cancer in the European screening cohort, $\mathbf{c}$ predicting any prostate cancer in the European clinical cohort, $\mathbf{d}$ predicting high-grade prostate cancer in the European clinical cohort, e predicting any prostate cancer in the
EU Clinical

(c) Any PCa

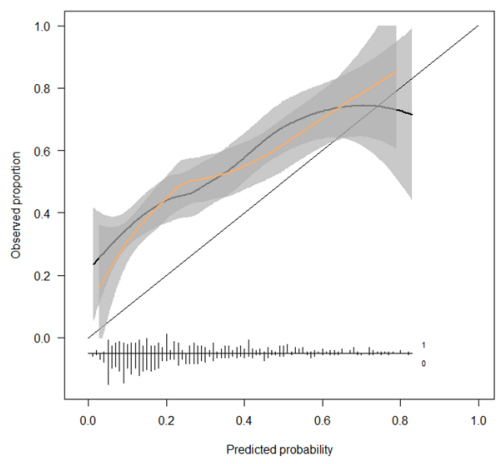

(d) HGPCa

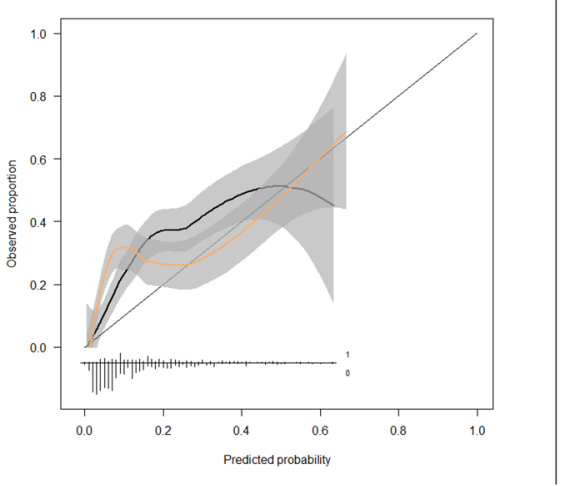

\section{Chinese}

(e) Any PCa

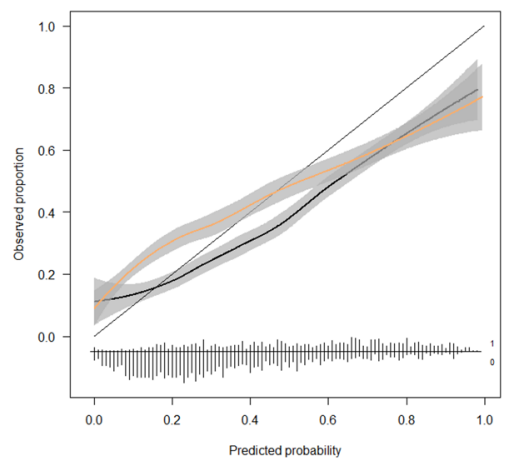

(f) HGPCa

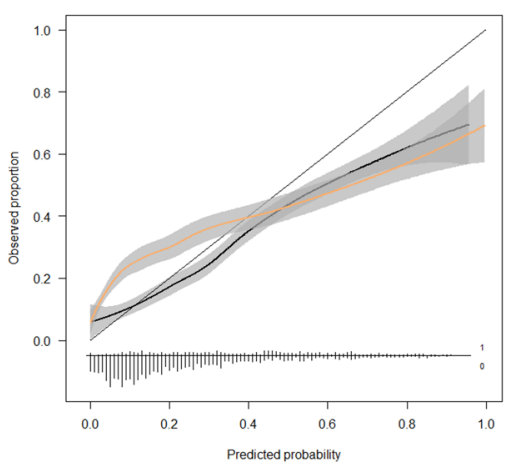
Chinese clinical cohort. The yellow line is for A-ERSPC-RC3 and the black line is for the CPCC-RC. $P C a$ prostate cancer, $H G P C a$ highgrade prostate cancer 

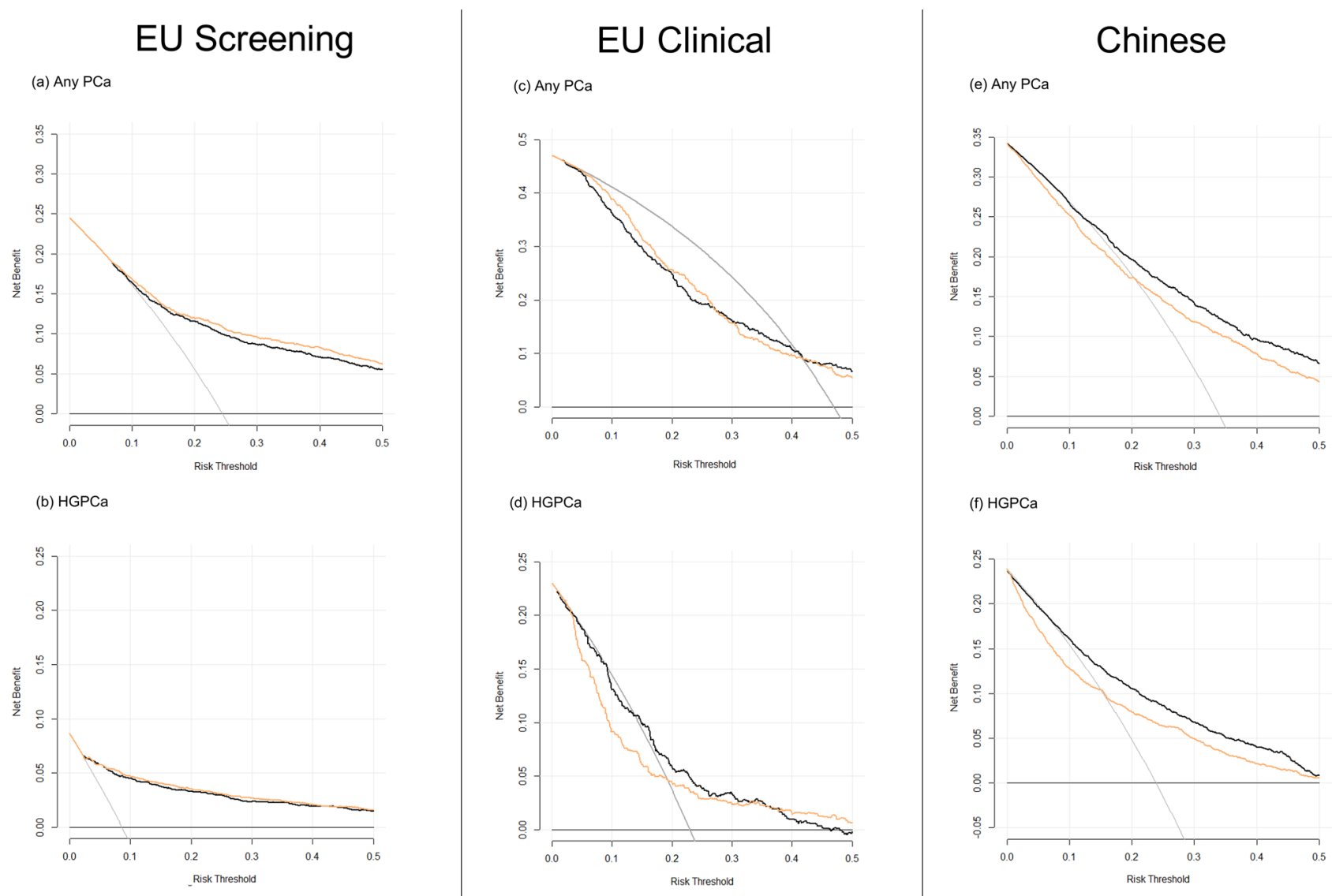

CPCC-RC

A-ERSPC-RC3

Fig. 2 Decision curve analysis for a any prostate cancer in the European screening cohort, b high-grade prostate cancer in the European screening cohort, c any prostate cancer in the European clinical cohort, $\mathbf{d}$ high-grade prostate cancer in the European clinical cohort, e predicting any prostate cancer in the Chinese clinical cohort, $\mathbf{f}$ highgrade prostate cancer in the Chinese clinical cohort. The yellow line is for A-ERSPC-RC3 and the black line is for the CPCC-RC. $P C a$ prostate cancer, $\mathrm{HGPCa}$ high-grade prostate cancer differences between a population-based screening setting as compared to a referral population [17]. In addition, the number of cores, age and the PSA levels differ between the cohort on which the A-ERSPC-RC was updated (Hong Kong origin). These differences could explain the underestimation of the A-ERSPC-RC. In comparison with the original ERSPC-RC, Zhu, et al. found that it overestimated the probability of PCa and HGPCa by approximately 20\% [9]. Yoon et al. validated the ERSPC-RC in a Korean cohort and showed the predicted probabilities were nearly $20 \%$ higher than the actual rate [8]. The over-estimation of the original ERSPC-RC and under-estimation of the A-ERSPC-RC indicates that the A-ERSPC-RC could need a more regionspecific adaptation or addition of other relevant predictors, e.g. age. When evaluating RCs in other settings, the risk factors distribution in relation to the outcome should be taken into consideration: i.e. the case mix of a cohort. This could explain the differences in the predictive performance of the tested RCs. For example, the PSA-PCa ratio might contribute to a good predictive capability in the Chinese cohort and the European screening cohort, while it could be the reason for a poor prediction in the EU clinical cohorts.

For the CPCC-RC, the predictive performance was good in the independent Chinese cohort as well as in the European screening cohort, but not in the European clinical cohort. This suggests that the CPCC-RC is applicable outside its development setting in Asia, but as expected less suitable in the European clinical setting with in general a higher prevalence of $\mathrm{PCa}$ and significant $\mathrm{PCa}$. This observation is confirmed by Ankerts et al. in where an internationally validated risk calculator for patients in North America and Europe was developed [17]. They found a substantial deviation between 
Table 3 Biopsy reduction, delayed prostate cancers diagnosis and net true positives using the Chinese Prostate Cancer Consortium Risk Calculator and the Asian adapted Rotterdam European Randomized
Study of Screening for Prostate Cancer Risk Calculator 3 compared to biopsy all in 1000 Chinese men

\begin{tabular}{|c|c|c|c|c|c|c|c|}
\hline \multirow{2}{*}{$\begin{array}{l}\text { Threshold } \\
\text { Clinical significant } \\
\text { PCa }\end{array}$} & \multicolumn{2}{|c|}{ Number of biopsies } & \multicolumn{2}{|c|}{ Number of indolent cancers } & \multicolumn{2}{|c|}{ Number of high-grade cancers } & \multirow{2}{*}{$\begin{array}{l}\text { Additional net true } \\
\text { reduction of biopsies } \\
\text { compared to ERSPC } \\
\text { strategy } \\
(\mathrm{G}) \times 1000 \text { men }\end{array}$} \\
\hline & (A) Performed & $\begin{array}{l}(\mathrm{B}) \\
\text { Reduced (\% } \\
\text { total A) }\end{array}$ & (C) Detected (\% A) & $\begin{array}{l}\text { (D) Not } \\
\text { detected } \\
(\% \mathrm{~B})\end{array}$ & (E) Detected (\% A) & $\begin{array}{l}(\mathrm{F}) \text { Missed } \\
\text { diagnosis } \\
(\% \mathrm{~B})\end{array}$ & \\
\hline $\begin{array}{l}\text { Biopsy all } \\
5 \%\end{array}$ & 1000 & 0 & 342 & 0 & $239(24)$ & 0 & \\
\hline CPCC-RC & 900 & $100(10)$ & $330(37)$ & $12(12)$ & $231(26)$ & $8(8)$ & 0.7 \\
\hline A-ERSPC-RC3 & 873 & $127(13)$ & $335(38)$ & $7(6)$ & $229(26)$ & $10(8)$ & - \\
\hline $10 \%$ & & & & & & & \\
\hline CPCC-RC & 752 & $248(25)$ & $310(41)$ & $32(13)$ & $220(29)$ & $19(8)$ & 6.8 \\
\hline A-ERSPC-RC3 & 725 & $275(28)$ & $325(45)$ & $17(6)$ & $211(29)$ & $28(10)$ & - \\
\hline $15 \%$ & & & & & & & \\
\hline CPCC-RC & 612 & $388(39)$ & $285(47)$ & $57(15)$ & $202(33)$ & $37(10)$ & 7.4 \\
\hline A-ERSPC-RC3 & 604 & $396(40)$ & $313(52)$ & $29(7)$ & $194(32)$ & $45(11)$ & - \\
\hline $20 \%$ & & & & & & & \\
\hline CPCC-RC & 512 & $488(49)$ & $258(50)$ & 84 (17) & $184(36)$ & $55(11)$ & 3.9 \\
\hline A-ERSPC-RC3 & 515 & $485(49)$ & $295(57)$ & $47(10)$ & $182(35)$ & $57(12)$ & - \\
\hline
\end{tabular}

CPCC-RC Chinese Prostate Cancer Consortium Risk Calculator, A-ERSPC-RC3 Asian adapted Rotterdam European Randomized Study of Screening for Prostate Cancer Risk Calculator 3

the predicted risk and the actual risk when applying the Prostate Cancer Prevention Trial risk calculator (developed in the US) to data from European centers. Using the data from eight North American medical centers, a new comprehensive risk prediction model was established and validated in three European centers. Less deviation was found with the newly developed Prostate Biopsy Collaborative Group model. Although they did not look at predictive performance in an Asian cohort, the message was similar to ours: external validation and recalibration are necessary before implementing RCs in daily clinical practice. Similarly, this study indicated the inapplicability of a European risk calculator in an Asian population. The CPCC-RC has shown to be well-calibrated in an East Asian population [10]. There are several other RCs in East Asian populations, such as Chinese [18], Korean [19, 20], and Japanese RCs [21]. Head-tohead comparison is required to compare these several RCs in clinical utility.

Development of numerous RCs is not helping the implementation of these useful tools. Instead, adapting existing RCs known to have good discriminative capability or constructing an internationally based risk calculator taking into account of ethnicity and heterogeneity between populations would be the way to go to optimize prostate cancer prediction on biopsy worldwide. The additional benefit of an international risk calculator would be a uniform patient selection for PCa, making it easier to compare study outcomes. In addition, existing and externally validated (international) RCs must be constantly updated with new findings that potentially can improve predictive capability. Multiparametric magnetic resonance imagining and novel biomarkers as four kallikrein, Prostate Cancer Antigen (PCA3) and Prostate Health Index (PHI) are promising additions in RCs that are used to select men with an elevated risk of having HGPCa and should be incorporated in multivariable prediction tools to analyze their potential improvement of the clinical utility [22, 23]. Importantly, MRI has evolved as a powerful test in the diagnostic situation [24], which has been recommended by the latest EAU guidelines for initial biopsy setting.

There are several limitations to our study. First, currently, ISUP classification was adopted in the assessment of risk for PCa RCs just as our previous studies which incorporate ISUP classifications [7], however, we did not apply ISUP standards due to the lack of the detailed Gleason scores. Further validation of ISUP classifications should be applied. Second, the three cohorts of patients differ in testing methodology, mainly in the nature of the participants and the indication for biopsy. It is important to note that the Chinese cohort is limited to men referred for a prostate cancer evaluation and do not reflect the general (screening) population, thus there is sensible risk of selection in the clinical cohort. Furthermore, the design to external validation of the RCs is retrospective; however, the data is prospectively collected for 
the European screening cohort. In the Chinese cohort there were some missing variables, but due to imputation could still be used for analysis. In general, it has been shown in many studies that instead of using a PSA cut-off for patient selection risk calculators should be used, as these tools can reduce one-third of the unnecessary biopsies [7].

\section{Conclusion}

The Asian-adapted ERSPC-RC3 performed well in a contemporary Chinese multi-center clinical cohort. The CPCC$\mathrm{RC}$ performed well in a European PSA based screening setting, but less in a multi-center European clinical cohort. Both RCs reduce unnecessary biopsies compared to a PSAdecision-based strategy. External validation and calibration are very necessary before implementing RCs.

Acknowledgements This study is supported by National Natural Science Foundation Youth Project (Rui Chen, 81702514). We give our sincere gratitude to Chinese Prostate Cancer Consortium, and Prof. Wei Xue (Renji Hospital, Shanghai Jiao Tong University, School of Medicine), Prof. Liping Xie (First Affiliated Hospital, School of Medicine, Zhejiang University, Hangzhou), Prof. Liqun Zhou, (Peking University First Hospital, Institute of Urology, Peking University, National Urological Cancer Center, Beijing) for collecting the biopsy data.

Author contributions RC: protocol/project development, data collection or management, manuscript writing/editing. JMV: protocol/project development, data collection or management, data analysis, manuscript writing/editing. YY: data collection or management, manuscript editing. ZS: data collection or management, manuscript editing. YS: protocol/project development. MJR: protocol/project development.

\section{Compliance with ethical standards}

Conflict of interest The authors have no potential conflicts of interest to disclose.

Research involving human participants This study is approved by Ethical review board of Changhai Hospital.

\section{References}

1. Siegel RL, Miller KD, Jemal A (2015) Cancer statistics, 2015. CA Cancer J Clin 65:5

2. Torre LA, Bray F, Siegel RL et al (2015) Global cancer statistics, 2012. CA Cancer J Clin 65:87

3. Chen R, Ren S, Yiu MK et al (2014) Prostate cancer in Asia: a collaborative report. Asian Journal of Urology 1:15

4. Kitagawa Y, Namiki M (2015) Prostate-specific antigen-based population screening for prostate cancer: current status in Japan and future perspective in Asia. Asian J Androl 17:475

5. Roobol MJ, Steyerberg EW, Kranse R et al (2010) A risk-based strategy improves prostate-specific antigen-driven detection of prostate cancer. Eur Urol 57:79

6. Chen R, Sjoberg DD, Huang Y et al (2017) Prostate specific antigen and prostate cancer in Chinese men undergoing initial prostate biopsies compared with western cohorts: data from the PBCG and CPCC. J Urol 197:90-96
7. Roobol MJ, Verbeek JFM, van der Kwast $\mathrm{T}$ et al (2017) Improving the Rotterdam European Randomized Study of Screening for Prostate Cancer Risk Calculator for Initial Prostate Biopsy by Incorporating the 2014 International Society of Urological Pathology Gleason Grading and Cribriform growth. Eur Urol 72:45

8. Yoon DK, Park JY, Yoon S et al (2012) Can the prostate risk calculator based on Western population be applied to Asian population? Prostate 72:721

9. Zhu Y, Wang JY, Shen YJ et al (2012) External validation of the Prostate Cancer Prevention Trial and the European Randomized Study of Screening for Prostate Cancer risk calculators in a Chinese cohort. Asian J Androl 14:738

10. Chen R, Xie L, Xue W et al (2016) Development and external multicenter validation of Chinese Prostate Cancer Consortium prostate cancer risk calculator for initial prostate biopsy. Urol Oncol 34:416 e1

11. Chiu PK, Roobol MJ, Nieboer D et al (2017) Adaptation and external validation of the European randomised study of screening for prostate cancer risk calculator for the Chinese population. Prostate Cancer Prostatic Dis 20:99

12. van Buuren S, Groothuis-Oudshoorn CGM (2011) Multivariate imputation by chained equations in R. J Stat Softw 45:1

13. Roobol MJ, van Vugt HA, Loeb S et al (2012) Prediction of prostate cancer risk: the role of prostate volume and digital rectal examination in the ERSPC risk calculators. Eur Urol 61:577

14. Gleason DF, Mellinger GT (1974) Prediction of prognosis for prostatic adenocarcinoma by combined histological grading and clinical staging. J Urol 111:58

15. Humphrey PA (2004) Gleason grading and prognostic factors in carcinoma of the prostate. Mod Pathol 17:292

16. Chiu PK, Roobol MJ, Nieboer D et al (2016) Adaptation and external validation of the European randomised study of screening for prostate cancer risk calculator for the Chinese population. Prostate Cancer Prostatic Dis 20:99-104

17. Ankerst DP, Straubinger J, Selig K et al (2018) A Contemporary prostate biopsy risk calculator based on multiple heterogeneous cohorts. Eur Urol 74:197

18. Wu YS, Zhang N, Liu SH et al (2016) The Huashan risk calculators performed better in prediction of prostate cancer in Chinese population: a training study followed by a validation study. Asian J Androl 18:925

19. Park JY, Yoon S, Park MS et al (2017) Development and external validation of the Korean Prostate Cancer Risk Calculator for HighGrade Prostate Cancer: comparison with two Western Risk Calculators in an Asian Cohort. PLoS ONE 12:e0168917

20. Park JY, Yoon S, Park MS et al (2011) Initial biopsy outcome prediction in Korean patients-comparison of a noble web-based Korean prostate cancer risk calculator versus prostate-specific antigen testing. J Korean Med Sci 26:85

21. Suzuki H, Komiya A, Kamiya N et al (2006) Development of a nomogram to predict probability of positive initial prostate biopsy among Japanese patients. Urology 67:131

22. Loeb S, Bruinsma SM, Nicholson J et al (2015) Active surveillance for prostate cancer: a systematic review of clinicopathologic variables and biomarkers for risk stratification. Eur Urol 67:619

23. Vedder MM, de Bekker-Grob EW, Lilja HG et al (2014) The added value of percentage of free to total prostate-specific antigen, PCA3, and a kallikrein panel to the ERSPC risk calculator for prostate cancer in prescreened men. Eur Urol 66:1109

24. Osses DF, Roobol MJ, Schoots IG (2019) Prediction medicine: biomarkers, risk calculators and magnetic resonance imaging as risk stratification tools in prostate cancer diagnosis. Int J Mol Sci. https ://doi.org/10.3390/ijms20071637

Publisher's Note Springer Nature remains neutral with regard to jurisdictional claims in published maps and institutional affiliations. 